\title{
On partial inverse operations in the class of preradicals of modules
}

\author{
Ion Jardan
}

\begin{abstract}
In the present work two partial operations in the class of preradicals $\mathbb{P R}$ of the category $R$-Mod of left $R$-modules are defined and investigated. They are inverse operations for product with respect to meet and coproduct with respect to join. The criteria of existence of such operations are indicated. Main properties of this operation and relations with the lattice operations in $\mathbb{P R}$ are shown. Some particular cases are mentioned.
\end{abstract}

\section{Introduction and preliminary facts}

This work is devoted to the theory of radicals of modules ([1]-[5]) and contains the definitions and the investigations of two new operations in the class of preradicals of a module category.

Let $R$ be a ring with unity and $R$-Mod be the category of unitary left $R$-modules. We remind that a preradical $r$ of $R$-Mod is a subfunctor of identity functor of $R$-Mod, i.e. $r$ associates to every module $M \in R$-Mod a submodule $r(M) \subseteq M$ such that $f(r(M)) \subseteq r\left(M^{\prime}\right)$ for every $R$-morphism $f: M \rightarrow M^{\prime}$.

We denote by $\mathbb{P R}$ the class of all preradicals of the category $R$-Mod. In this class four operation are defined [1]:

Key Words: ring, module, lattice, preradical, join, meet, product, coproduct.

2010 Mathematics Subject Classification: 16D90, 16S90.

Received: 06.07.2018

Accepted: 05.09.2018 
1) the meet $\underset{\alpha \in \mathfrak{A}}{\wedge} r_{\alpha}$ of a family of preradicals $\left\{r_{\alpha}\right\}_{\alpha \in \mathfrak{A}}$ :

$$
\left(\hat{\alpha \in \mathfrak{A}}_{\alpha} r_{\alpha}\right)(M) \stackrel{\text { def }}{=} \bigcap_{\alpha \in \mathfrak{A}} r_{\alpha}(M), M \in R \text {-Mod; }
$$

2) the join $\underset{\alpha \in \mathfrak{A}}{\vee} r_{\alpha}$ of a family of preradicals $\left\{r_{\alpha}\right\}_{\alpha \in \mathfrak{A}}$ :

$$
\left(\vee_{\alpha \in \mathfrak{A}} r_{\alpha}\right)(M) \stackrel{\text { def }}{=} \sum_{\alpha \in \mathfrak{A}} r_{\alpha}(M), M \in R \text {-Mod; }
$$

3) the product $r \cdot s$ of preradicals $r, s \in \mathbb{P} \mathbb{R}$ :

$$
(r \cdot s)(M) \stackrel{\text { def }}{=} r(s(M)), M \in R \text {-Mod ; }
$$

4) the coproduct $r \# s$ of preradicals $r, s \in \mathbb{P}$ :

$$
[(r \# s)(M)] / s(M) \stackrel{\text { def }}{=} r(M / s(M)), M \in R \text {-Mod. }
$$

In the class $\mathbb{P R}$ the partial order relation " $\leq$ " is defined by the rule:

$$
r_{1} \leq r_{2} \stackrel{\text { def }}{\Leftrightarrow} r_{1}(M) \subseteq r_{2}(M) \text { for every } M \in R \text {-Mod. }
$$

The class $\mathbb{P} \mathbb{R}$ is a large complete lattice with respect to the operations of meet and join.

We remark that in the book [1] the coproduct is denoted by $(r: s)$ and is defined by the rule $[(r: s)(M)] / r(M)=s(M / r(M))$, so $(r \# s)=(s: r)$.

The following properties of distributivity hold ([1]-[5]):

(1) $\left(\wedge r_{\alpha}\right) \cdot s=\wedge\left(r_{\alpha} \cdot s\right)$;

(2) $\left(\vee r_{\alpha}\right) \cdot s=\vee\left(r_{\alpha} \cdot s\right)$;

(3) $\left(\wedge r_{\alpha}\right) \# s=\wedge\left(r_{\alpha} \# s\right)$;

(4) $\left(\vee r_{\alpha}\right) \# s=\vee\left(r_{\alpha} \# s\right)$

for every family $\left\{r_{\alpha}\right\}_{\alpha \in \mathfrak{A}} \subseteq \mathbb{P} \mathbb{R}$ and $s \in \mathbb{P}$.

Using these relations some new inverse operations can be defined in the class $\mathbb{P} R$. Two of them, the left quotient with respect to join and the left coquotient with respect to meet, have been defined and investigated in [11] and [12]. In this work we will study other two inverse operations, namely, the left quotient with respect to meet and the left coquotient with respect to join. Similar questions are discussed in [8]-[10].

Now we remind the principal types of preradicals. A preradical $r \in \mathbb{P}$ is called:

- idempotent preradical, if $r(r(M))=r(M)$ for every $M \in R$-Mod (or if $r \cdot r=r)$;

- radical, if $r(M / r(M))=0$ for every $M \in R$-Mod (or if $r \# r=r$ );

- idempotent radical, if both previous conditions are fulfilled;

- prime, if $r \neq 1$ and for any $t_{1}, t_{2} \in \mathbb{P} \mathbb{R}, t_{1} \cdot t_{2} \leq r$ implies $t_{1} \leq r$ or $t_{2} \leq r[6]$

- coprime, if $r \neq 0$ and for any $t_{1}, t_{2} \in \mathbb{P}, t_{1} \# t_{2} \geq r$ implies $t_{1} \geq r$ or $t_{2} \geq r[7]$; 
- $\wedge$-prime, if for any $t_{1}, t_{2} \in \mathbb{P} R, t_{1} \wedge t_{2} \leq r$ implies $t_{1} \leq r$ or $t_{2} \leq r$ $[6]$

- $\vee$-coprime, if for any $t_{1}, t_{2} \in \mathbb{P} \mathbb{R}, t_{1} \vee t_{2} \geq r$ implies $t_{1} \geq r$ or $t_{2} \geq r$ $[7]$

- irreducible, if for any $t_{1}, t_{2} \in \mathbb{P R}, t_{1} \wedge t_{2}=r$ implies $t_{1}=r$ or $t_{2}=r$ $[6]$

- coirreducible, if for any $t_{1}, t_{2} \in \mathbb{P R}, t_{1} \vee t_{2}=r$ implies $t_{1}=r$ or $t_{2}=r[7]$.

The operations of meet and join are commutative and associative, while the operations of product and coproduct are associative. By means of these operations four preradicals are obtained which are arranged in the following order:

for every $r, s \in \mathbb{P} \mathbb{R}$.

$$
r \cdot s \leq r \wedge s \leq r \vee s \leq r \# s
$$

During this work we will use the following facts and notions from general theory of preradicals (see $[1]-[7]$ ).

Lemma 1.1. (Monotony of the product) For any $s_{1}, s_{2} \in \mathbb{P R}, s_{1} \leq s_{2}$ implies that $r \cdot s_{1} \leq r \cdot s_{2}$ and $s_{1} \cdot r \leq s_{2} \cdot r$ for every $r \in \mathbb{P}$.

Lemma 1.2. (Monotony of the coproduct) For any $s_{1}, s_{2} \in \mathbb{P R}, s_{1} \leq s_{2}$ implies that $r \# s_{1} \leq r \# s_{2}$ and $s_{1} \# r \leq s_{2} \# r$ for every $r \in \mathbb{P}$.

Lemma 1.3. For every $r, s, t \in \mathbb{P}$ we have:

1) $(r \cdot s) \# t \geq(r \# t) \cdot(s \# t)$;

2) $(r \# s) \cdot t \leq(r \cdot t) \#(s \cdot t)$.

Definition 1.1. The equalizer of preradical $r$ is the preradical

$$
e(r)=\wedge\left\{r_{\alpha} \in \mathbb{P} \mathbb{R} \mid r_{\alpha} \cdot r=r\right\} .
$$

Definition 1.2. The co-equalizer of preradical $r$ is the preradical

$$
c(r)=\vee\left\{r_{\alpha} \in \mathbb{P} \mathbb{R} \mid r_{\alpha} \# r=r\right\} .
$$

\section{Left quotient with respect to meet}

Now we introduce and investigate the inverse operation of product with respect to meet in the class of preradicals $\mathbb{P R}$ of category $R$-Mod.

Definition 2.1. Let $r, s \in \mathbb{P} \mathbb{R}$. The left quotient with respect to meet of $r$ by $s$ is defined as the least preradical among $r_{\alpha} \in \mathbb{P} \mathbb{R}$ with the property $r_{\alpha} \cdot s \geq r$. We denote this preradical by $r \wedge s$. 
We will call $r$ the numerator and $s$ the denominator of the quotient $r \% s$.

Now we will study question on the existence of the left quotient with respect to meet.

Lemma 2.1. Let $r, s \in \mathbb{P R}$. The left quotient $r \% s$ of $r$ by $s$ with respect to meet exists if and only if $r \leq s$ and it can be presented in the form $r \wedge . s=\wedge\left\{r_{\alpha} \in \mathbb{P} \mathbb{R} \mid r_{\alpha} \cdot s \geq r\right\}$.

Proof. $(\Rightarrow)$ If the left quotient $r \% s$ exists, then there exists $r_{\alpha} \in \mathbb{P} \mathbb{R}$ such that $r_{\alpha} \cdot s \geq r$. Since $1 \geq r_{\alpha}$, from the monotony of product we have $1 \cdot s \geq r_{\alpha} \cdot s$, therefore $s \geq r$.

$(\Leftarrow)$ Let $r \leq s$. Then $1 \cdot s=s \geq r$, therefore the family of preradicals $\left\{r_{\alpha} \in \mathbb{P} \mathbb{R} \mid r_{\alpha} \cdot s \geq r\right\}$ is not empty. So we can consider the preradical $\wedge\left\{r_{\alpha} \in \mathbb{P} \mathbb{R} \mid r_{\alpha} \cdot s \geq r\right\}$, for which by distributivity of product relative to

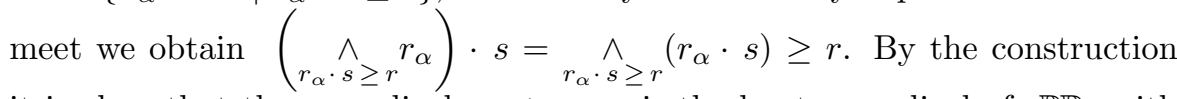

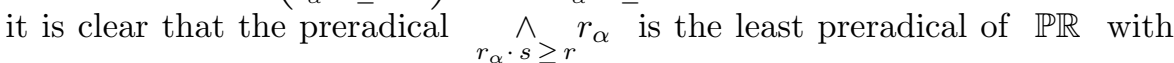
property $r_{\alpha} \cdot s \geq r$. Therefore we have $r \wedge s=\wedge\left\{r_{\alpha} \in \mathbb{P} \mathbb{R} \mid r_{\alpha} \cdot s \geq r\right\}$.

Moreover, by the proof of Lemma 2.1 we have that $(r \% s) \cdot s \geq r$, what we will often use further.

Lemma 2.2. Let $r, s \in \mathbb{P}$ and $r \leq s$. Then $r \% s \geq r$.

Proof. The condition $r \leq s$ ensures the existence of the left quotient $r \wedge s$. Since $r \% s \geq(r \wedge s) \cdot s$, by the definition of the left quotient $(r \wedge s) \cdot s \geq r$, it follows that $r \wedge s \geq r$.

The next two statements show the concordance of the left quotient $r \% s$ with the order relation $(\leq)$ of $\mathbb{P R}$.

Proposition 2.3. (Monotony in the numerator) Let $r_{1}, r_{2} \in \mathbb{P}$ and $r_{1} \leq r_{2}$. Then for every preradical $s \geq r_{2}$ we have $r_{1} \wedge s \leq r_{2} \% s$.

Proof. By Lemma 2.1 there exist the left quotients $r_{1} \wedge, s, r_{2} \% s$ and $r_{1} \wedge . s=\wedge\left\{r_{\alpha} \in \mathbb{P} \mathbb{R} \mid r_{\alpha} \cdot s \geq r_{1}\right\}, r_{2} \% s=\wedge\left\{r_{\beta}^{\prime} \in \mathbb{P} \mathbb{R} \mid r_{\beta}^{\prime} \cdot s \geq r_{2}\right\}$.

Let $r_{1} \leq r_{2}$ and $r_{\beta}^{\prime} \cdot s \geq r_{2}$. Then $r_{\beta}^{\prime} \cdot s \geq r_{1}$, so each $r_{\beta}^{\prime}$ is one of preradicals $r_{\alpha}$. Therefore $\wedge\left\{r_{\alpha} \in \mathbb{P} \mathbb{R} \mid r_{\alpha} \cdot s \geq r_{1}\right\} \leq \wedge\left\{r_{\beta}^{\prime} \in \mathbb{P} \mathbb{R} \mid r_{\beta}^{\prime} \cdot s \geq r_{2}\right\}$, i.e. $r_{1} \% s \leq r_{2} \% s$.

Proposition 2.4. (Antimonotony in the denominator) Let $s_{1}, s_{2} \in \mathbb{P} \mathbb{R}$ and $s_{1} \leq s_{2}$. Then for every preradical $r \leq s_{1}$ we have $r \% s_{1} \geq r \wedge s_{2}$. 
Proof. By Lemma 2.1 there exist the left quotients $r \% s_{1}, r \% s_{2}$ and $r \wedge s_{1}=\wedge\left\{r_{\alpha} \in \mathbb{P R} \mid r_{\alpha} \cdot s_{1} \geq r\right\}$ and $r \wedge s_{2}=\wedge\left\{r_{\beta}^{\prime} \in \mathbb{P} \mathbb{R} \mid r_{\beta}^{\prime} \cdot s_{2} \geq r\right\}$.

Let $r_{\alpha} \cdot s_{1} \geq r$. If $s_{1} \leq s_{2}$, then from the monotony of product we have $r_{\alpha} \cdot s_{1} \leq r_{\alpha} \cdot s_{2}$, but $r_{\alpha} \cdot s_{1} \geq r$, therefore $r_{\alpha} \cdot s_{2} \geq r$. So each preradical $r_{\alpha}$ is one of preradicals $r_{\beta}^{\prime}$, what implies $\wedge\left\{r_{\alpha} \in \mathbb{P} \mathbb{R} \mid r_{\alpha} \cdot s_{1} \geq r\right\} \geq$ $\wedge\left\{r_{\beta}^{\prime} \in \mathbb{P} \mathbb{R} \mid r_{\beta}^{\prime} \cdot s_{2} \geq r\right\}$, i.e. $r \wedge s_{1} \geq r \% s_{2}$.

The following fact is very useful for the further investigations.

Proposition 2.5. Let $r, s \in \mathbb{P} \mathbb{R}$ and $r \leq s$. Then for every $t \in \mathbb{P}$ we have:

$$
r \leq t \cdot s \Leftrightarrow r \wedge s \leq t
$$

Proof. By Lemma 2.1 there exists the left quotient $r \wedge s$ and $r \wedge s=$ $\wedge\left\{r_{\alpha} \in \mathbb{P} \mathbb{R} \mid r_{\alpha} \cdot s \geq r\right\}$.

$(\Rightarrow)$ Let $t \cdot s \geq r$. Then $t$ is one of preradicals $r_{\alpha}$, therefore $t \geq$ $\wedge\left\{r_{\alpha} \in \mathbb{P} \mathbb{R} \mid r_{\alpha} \cdot s \geq r\right\}$, i.e. $t \geq r \wedge s$.

$(\Leftarrow)$ Let $r \% s \leq t$. From the monotony of product $(r \wedge s) \cdot s \leq t \cdot s$, but by the definition of the left quotient we have $(r \% s) \cdot s \geq r$, therefore $t \cdot s \geq r$.

In continuation we show some properties of the studied operation.

Proposition 2.6. For every preradicals $r, s \in \mathbb{P}$ we have:

$$
(r \cdot s) \wedge s \leq r .
$$

Proof. Since $r \cdot s \leq s$, then there exists the left quotient $(r \cdot s) \wedge s$ and from Lemma $2.1(r \cdot s) \wedge, s=\wedge\left\{t_{\alpha} \in \mathbb{P R} \mid t_{\alpha} \cdot s \geq r \cdot s\right\}$. Because $r \cdot s \geq r \cdot s$, the preradical $r$ is one of preradicals $t_{\alpha}$, therefore $r \geq$ $\wedge\left\{t_{\alpha} \in \mathbb{P} \mathbb{R} \mid t_{\alpha} \cdot s \geq r \cdot s\right\}$, i.e. $r \geq(r \cdot s) \wedge . s$.

Proposition 2.7. Let $r, s \in \mathbb{P} \mathbb{R}$. The following relations are true:

1) $(r \% s) \wedge t=r \%(t \cdot s)$ for any preradical $t$ with the property $t \cdot s \geq r$

2) $(r \cdot s) \wedge t \leq r \cdot(s \% t)$ for any preradical $t \geq s$.

Proof. 1) If $r \leq t \cdot s$, then there exists the left quotient $r \%(t \cdot s)$. In this case, since $t \cdot s \leq s$ we have $r \leq s$, so there exists the left quotient $r \% s$. Moreover, by Proposition $2.5 t \cdot s \geq r \Leftrightarrow r \wedge . s \leq t$, which ensures the existence of the left quotient $(r \% s) \% . t$. From Lemma 2.1 we have $r \%(t \cdot s)=$ $\wedge\left\{r_{\alpha} \in \mathbb{P} \mathbb{R} \mid r_{\alpha} \cdot(t \cdot s) \geq r\right\}$ and $(r \wedge s) \wedge t=\wedge\left\{t_{\beta} \in \mathbb{P} \mathbb{R} \mid t_{\beta} \cdot t \geq r \% s\right\}$.

$(\leq)$ Let $r_{\alpha} \cdot(t \cdot s) \geq r$. Since $\left(r_{\alpha} \cdot t\right) \cdot s=r_{\alpha} \cdot(t \cdot s)$ we have $\left(r_{\alpha} \cdot t\right) \cdot s \geq r$, but $r \% s$ is the least preradical with such property, so 
$r_{\alpha} \cdot t \geq r \% s$. Hence each $r_{\alpha}$ is one of preradicals $t_{\beta}$, which implies that for any $r_{\alpha}$ we have $r_{\alpha} \geq \wedge\left\{t_{\beta} \in \mathbb{P} \mid t_{\beta} \cdot t \geq r \wedge s\right\}$ for every $\alpha$, therefore $\wedge\left\{r_{\alpha} \in \mathbb{P} \mathbb{R} \mid r_{\alpha} \cdot(t \cdot s) \geq r\right\} \geq \wedge\left\{t_{\beta} \in \mathbb{P} \mathbb{R} \mid t_{\beta} \cdot t \geq r \wedge s\right\}$, i.e. $r \wedge(t \cdot s) \geq(r \wedge s) \wedge . t$.

$(\geq)$ Let $t_{\beta} \cdot t \geq r \% s$. Using the associativity and the monotony of product of preradicals we obtain $t_{\beta} \cdot(t \cdot s)=\left(t_{\beta} \cdot t\right) \cdot s \geq(r \% s) \cdot s$, by the definition of the left quotient $(r \wedge s) \cdot s \geq r$, so $t_{\beta} \cdot(t \cdot s) \geq$ $r$. This shows that each preradical $t_{\beta}$ is one of preradicals $r_{\alpha}$, therefore we have $\wedge\left\{t_{\beta} \in \mathbb{P} \mathbb{R} \mid t_{\beta} \cdot t \geq r \wedge s\right\} \geq \wedge\left\{r_{\alpha} \in \mathbb{P} \mathbb{R} \mid r_{\alpha} \cdot(t \cdot s) \geq r\right\}$, i.e $(r \wedge . s) \wedge t \geq r \wedge .(t \cdot s)$.

2) The relation $s \leq t$ ensures the existence of the left quotient $s \% t$ and since $r \cdot s \leq s$ we have $r \cdot s \leq t$, so there exists and the left quotient $(r \cdot s) \wedge t . t$.

By the definition of the left quotient $s \leq(s \% t) \cdot t$. Using the monotony and the property of associativity of product of preradicals it follows that $r$. $s \leq r \cdot[(s \% t) \cdot t]=[r \cdot(s \% t)] \cdot t$. Applying Proposition 2.5 for relation $r \cdot s \leq[r \cdot(s \% t)] \cdot t$ we obtain $(r \cdot s) \wedge t \leq r \cdot(s \% t)$.

Proposition 2.8. Let $r, s, t \in \mathbb{P}$ and $r \leq s$. Then the following relations hold:

1) $(r \% t) \wedge(s \% t) \leq r \wedge . s$ or $(r \% s) \cdot(s \% t) \geq r \wedge \cdot t$ for any preradical $t \geq s$;

2) $(r \cdot t) \%(s \cdot t) \leq r \% s$ for any preradical $t \in \mathbb{P} \mathbb{R}$.

Proof. 1) The conditions $r \leq s$ and $s \leq t$ ensure the existence of the left quotients $r \% s$ and $s \% t$. In this case, since $r \leq s$ and $s \leq t$ we have $r \leq t$, so there exists the left quotient $r \% t$. Moreover, since $r \leq s$, from the monotony of the left quotient it follows that $r \wedge t \leq s \% t$, which ensures the existence of the left quotient $(r \wedge t) \%(s \% t)$.

From Proposition 2.5 the relations of this statement are equivalent.

By the definition of the left quotient $r \leq(r \% s) \cdot s$ and $s \leq(s \% t) \cdot t$. Therefore, using the monotony and the associativity of product we obtain $r \leq$ $(r \wedge s) \cdot s \leq(r \wedge s) \cdot[(s \% t) \cdot t]=[(r \wedge s) \cdot(s \% t)] \cdot t$. Applying Proposition 2.5 for the relation $r \leq[(r \% s) \cdot(s \% t)] \cdot t$ we have $r \% t \leq(r \% s) \cdot(s \wedge t)$.

2) The condition $r \leq s$ ensures the existence of the left quotient $r \% s$. In this case, by the monotony of product we have $r \cdot t \leq s \cdot t$ for every $t \in \mathbb{P} \mathbb{R}$, so there exists the left quotient $(r \cdot t) \%(s \cdot t)$.

From Proposition 2.5 the relation of this statement is equivalent to the relation $r \cdot t \leq(r \wedge s) \cdot(s \cdot t)$.

By the definition of the left quotient $r \leq(r \wedge s) \cdot s$, therefore applying the monotony and the associativity of product we obtain $r \cdot t \leq[(r \wedge s) \cdot s] \cdot t=$ $(r \wedge s) \cdot(s \cdot t)$. 
Now we will discuss the question on the relations beetween the left quotient with respect to meet and the lattice operations of $\mathbb{P}$.

Proposition 2.9. (The left distributivity of the left quotient $r \wedge . s$ relative to join) Let $s \in \mathbb{P} \mathbb{R}$. Then for any family of preradicals $\left\{r_{\alpha} \in \mathbb{P} \mathbb{R} \mid r_{\alpha} \leq s, \alpha \in \mathfrak{A}\right\}$ the following relation holds:

$$
\left(\bigvee_{\alpha \in \mathfrak{A}} r_{\alpha}\right) \% s=\bigvee_{\alpha \in \mathfrak{A}}\left(r_{\alpha} \% s\right) .
$$

Proof. The relations $r_{\alpha} \leq s, \alpha \in \mathfrak{A}$ ensure the existence of the left quotients $r_{\alpha} \% s, \alpha \in \mathfrak{A}$. But in this case $\underset{\alpha \in \mathfrak{A}}{\vee} r_{\alpha} \leq s$, so there exists the left quotient $\left(\underset{\alpha \in \mathfrak{A}}{\vee} r_{\alpha}\right) \% s$.

( $\leq)$ By the definition of the left quotient $r_{\alpha} \leq\left(r_{\alpha} \% s\right) \cdot s$ for all $\alpha \in \mathfrak{A}$, which implies that $\underset{\alpha \in \mathfrak{A}}{\vee} r_{\alpha} \leq \bigvee_{\alpha \in \mathfrak{A}}\left[\left(r_{\alpha} \% s\right) \cdot s\right]$. Using the distributivity of product of preradicals relative to join $\underset{\alpha \in \mathfrak{A}}{\vee} r_{\alpha} \leq\left[\underset{\alpha \in \mathfrak{A}}{\vee}\left(r_{\alpha} \% s\right)\right] \cdot s$. Applying Proposition 2.5 we obtain $\left(\underset{\alpha \in \mathfrak{A}}{\vee} r_{\alpha}\right) \wedge s \leq \underset{\alpha \in \mathfrak{A}}{\vee}\left(r_{\alpha} \% s\right)$.

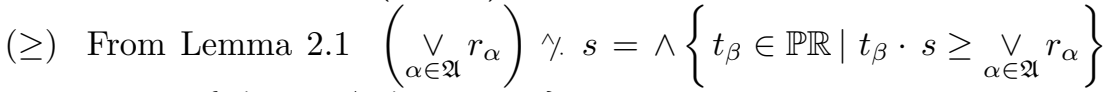
and $r_{\alpha} \% s=\wedge\left\{r_{\gamma}^{\prime} \in \mathbb{P} \mathbb{R} \mid r_{\gamma}^{\prime} \cdot s \geq r_{\alpha}\right\}$.

Let $t_{\beta} \cdot s \geq \underset{\alpha \in \mathfrak{A}}{\vee} r_{\alpha}$. Since $\underset{\alpha \in \mathfrak{A}}{\vee} r_{\alpha} \geq r_{\alpha}$ for every $\alpha \in \mathfrak{A}$ we have $t_{\beta} \cdot s \geq r_{\alpha}$, hence each preradical $t_{\beta}$ is one of preradicals $r_{\gamma}^{\prime}$. Therefore $\wedge\left\{t_{\beta} \in \mathbb{P R} \mid t_{\beta} \cdot s \geq \underset{\alpha \in \mathfrak{A}}{\vee} r_{\alpha}\right\} \geq \wedge\left\{r_{\gamma}^{\prime} \in \mathbb{P} \mathbb{R} \mid r_{\gamma}^{\prime} \cdot s \geq r_{\alpha}\right\} \quad$ for every $\alpha \in \mathfrak{A}$, i.e. $\left(\underset{\alpha \in \mathfrak{A}}{\vee} r_{\alpha}\right) \% s \geq r_{\alpha} \% s$ for every $\alpha \in \mathfrak{A}$, which implies that $\left(\underset{\alpha \in \mathfrak{A}}{\vee} r_{\alpha}\right) \wedge s \geq \underset{\alpha \in \mathfrak{A}}{\vee}\left(r_{\alpha} \% s\right)$

Proposition 2.10. In the class $\mathbb{P R}$ the following relations are true:

1) $\left(\hat{\alpha \in \mathfrak{A}}_{\alpha} r_{\alpha}\right) \% s \leq \hat{\wedge}_{\alpha \in \mathfrak{A}}\left(r_{\alpha} \% s\right)$, when $r_{\alpha} \leq s$ for any $\alpha \in \mathfrak{A}$;

2) $r \varkappa\left(\hat{\alpha \in \mathfrak{A}}_{\alpha} s_{\alpha}\right) \geq \underset{\alpha \in \mathfrak{A}}{\vee}\left(r \% s_{\alpha}\right)$, when $r \leq s_{\alpha}$ for any $\alpha \in \mathfrak{A}$;

3) $r \%\left(\vee_{\alpha \in \mathfrak{A}} s_{\alpha}\right) \leq \underset{\alpha \in \mathfrak{A}}{\wedge}\left(r \% s_{\alpha}\right)$, when $r \leq s_{\alpha}$ for any $\alpha \in \mathfrak{A}$

Proof. 1) The conditions $r_{\alpha} \leq s, \quad \alpha \in \mathfrak{A}$ ensure the existence of the left 
quotients $r_{\alpha} \% s, \alpha \in \mathfrak{A}$. But in this case $\bigwedge_{\alpha \in \mathfrak{A}} r_{\alpha} \leq s$, so there exists the left quotient $\left(\hat{\alpha}_{\alpha \in \mathfrak{A}} r_{\alpha}\right) \% s$.

By the definition of the left quotient we have $r_{\alpha} \leq\left(r_{\alpha} \% s\right) \cdot s$ for any $\alpha \in \mathfrak{A}$, which implies that $\bigwedge_{\alpha \in \mathfrak{A}} r_{\alpha} \leq \bigwedge_{\alpha \in \mathfrak{A}}\left[\left(r_{\alpha} \% s\right) \cdot s\right]$. Applying the distributivity of product of preradicals relative to meet it follows that $\bigwedge_{\alpha \in \mathfrak{A}} r_{\alpha} \leq$ $\left.\bigwedge_{\alpha \in \mathfrak{A}}\left(r_{\alpha} \% s\right)\right] \cdot s$ and from Proposition 2.5 we obtain $\left(\bigwedge_{\alpha \in \mathfrak{A}} r_{\alpha}\right)^{\alpha \in \mathfrak{A}} \mathrm{s} \leq$ $\bigwedge_{\alpha \in \mathfrak{A}}\left(r_{\alpha} \wedge s\right)$.

2) The conditions $r \leq s_{\alpha}, \alpha \in \mathfrak{A}$ ensure the existence of the left quotients $r \varkappa s_{\alpha}, \quad \alpha \in \mathfrak{A}$. But in this case $r \leq \bigwedge_{\alpha \in \mathfrak{A}} s_{\alpha}$, which implies the existence of the left quotient $r \%\left(\bigwedge_{\alpha \in \mathfrak{A}}^{\wedge} s_{\alpha}\right)$.

For any $\alpha \in \mathfrak{A}$ we have $\bigwedge_{\alpha \in \mathfrak{A}} s_{\alpha} \leq s_{\alpha}$. Using Proposition 2.4 we obtain $r \%\left(\hat{\alpha \in \mathfrak{A}}_{\alpha} s_{\alpha}\right) \geq r \% s_{\alpha}$ for all $\alpha \in \mathfrak{A}$, therefore $r \%\left(\hat{\alpha}_{\alpha \in \mathfrak{A}} s_{\alpha}\right) \geq$ $\bigvee_{\alpha \in \mathfrak{A}}\left(r \% s_{\alpha}\right)$.

3) The conditions $r \leq s_{\alpha}, \alpha \in \mathfrak{A}$ ensure the existence of the left quotients $r \% s_{\alpha}, \quad \alpha \in \mathfrak{A}$. Moreover, in this case $r \leq \underset{\alpha \in \mathfrak{A}}{\vee} s_{\alpha}$, so there exists the left quotient $r \wedge\left(\underset{\alpha \in \mathfrak{A}}{\vee} s_{\alpha}\right)$.

For any $\alpha \in \mathfrak{A}$ we have $\underset{\alpha \in \mathfrak{A}}{\vee} s_{\alpha} \geq s_{\alpha}$. Using Proposition 2.4 we obtain $r \%\left(\vee_{\alpha \in \mathfrak{A}} s_{\alpha}\right) \leq r \% s_{\alpha}$ for all $\alpha \in \mathfrak{A}$, therefore $r \%\left(\underset{\alpha \in \mathfrak{A}}{\vee} s_{\alpha}\right) \leq$ $\bigwedge_{\alpha \in \mathfrak{A}}\left(r \wedge s_{\alpha}\right)$.

Now we will consider some particular cases of the left quotient $r \% s$.

Proposition 2.11. Let $r, s \in \mathbb{P} \mathbb{R}$. Then:

1) $r \% r=e(r)$ (see Definition 1.1);

2) $r \wedge .1=r$;

3) $0 \% s=0$.

Proof. Using the definition of the left quotient we obtain:

1) $r \% r=\wedge\left\{r_{\alpha} \in \mathbb{P} \mathbb{R} \mid r_{\alpha} \cdot r \geq r\right\}=\wedge\left\{r_{\alpha} \in \mathbb{P} \mathbb{R} \mid r_{\alpha} \cdot r=r\right\}=e(r)$;

2) $r \wedge 1=\wedge\left\{r_{\alpha} \in \mathbb{P} \mathbb{R} \mid r_{\alpha} \cdot 1 \geq r\right\}=\wedge\left\{r_{\alpha} \in \mathbb{P} \mathbb{R} \mid r_{\alpha} \geq r\right\}=r$;

3) $0 \% s=\wedge\left\{r_{\alpha} \in \mathbb{P} \mathbb{R} \mid r_{\alpha} \cdot s \geq 0\right\}=\wedge\left\{r_{\alpha} \mid r_{\alpha} \in \mathbb{P} \mathbb{R}\right\}=0$. 
By Proposition 2.11 we have the following particular cases:
(1) $0 \% 0=0$;
(2) $1 \% 1=1$.

Applying to the relation $r \leq s \leq 1$ the statement of Proposition 2.4, we obtain $r \% r \geq r \% s \geq r \% 1$, hence $r \leq r \wedge s \leq e(r)$.

Moreover, the distributivity of product of preradicals relative to meet implies $e(r) \cdot r=\left({ }_{r_{\alpha} \cdot r=r}^{\wedge} r_{\alpha}\right) \cdot r={ }_{r_{\alpha} \cdot r=r}^{\wedge}\left(r_{\alpha} \cdot r\right)=r$ for every $r \in \mathbb{P}$.

The following statement shows some properties of equalizer.

Proposition 2.12. Let $r, s \in \mathbb{P} \mathbb{R}$ and $r \leq s$. Then:

1) $e(r) \cdot(r \% s)=r \% s$;

2) $(r \% s) \cdot e(s)=r \wedge s$;

3) $(r \% s) \% e(s)=r \% s$.

Proof. The condition $r \leq s$ ensures the existence of the left quotient $r \wedge s$. 1) $e(r) \cdot(r \wedge s)=(r \wedge r) \cdot(r \wedge s)$. Applying Proposition 2.8(1) we obtain $(r \wedge r) \cdot(r \% s) \geq r \% s$, but $(r \% r) \cdot(r \% s) \leq r \% s$, so $e(r) \cdot(r \% s)=$ $r \wedge s$.

2) $(r \% s) \cdot e(s)=(r \% s) \cdot(s \% s)$. Applying Proposition 2.8(1) we obtain $(r \wedge s) \cdot(s \wedge s) \geq r \wedge s$, but $(r \wedge s) \cdot(s \wedge s) \leq r \wedge s$, so $(r \wedge s) \cdot e(s)=$ $r \wedge s$.

3) $(r \% s) \% e(s)=(r \% s) \%(s \% s)$. Using Proposition 2.8(1) we obtain $(r \wedge s) \wedge(s \wedge s) \leq r \wedge s$, but from Lemma $2.2(r \wedge s) \wedge(s \wedge s) \geq r \wedge s$, so we have $(r \wedge s) \wedge e(s)=r \wedge s$.

We will now consider the case of idempotent preradicals.

Remark 2.13. ([5]) For every preradical $r \in \mathbb{P} \mathbb{R}$ we have $e(r)$ is an idempotent preradical.

Proof. $e(r) \cdot e(r)=(r \% r) \cdot(r \% r)$. From Proposition 2.8(1) we have $(r \wedge r) \cdot(r \% r) \geq r \% r$, but $(r \wedge r) \cdot(r \% r) \leq r \% r$, hence $e(r) \cdot e(r)=e(r)$, i.e. $e(r)$ is an idempotent preradical.

Proposition 2.14. ([5]) The preradical $r \in \mathbb{P} \mathbb{R}$ is idempotent if and only if $e(r)=r$.

Proof. $\quad(\Rightarrow)$ By Definition $1.2 e(r)=\wedge\left\{r_{\alpha} \in \mathbb{P} \mathbb{R} \mid r_{\alpha} \cdot r=r\right\}$. If $r$ is an idempotent preradical, i.e. $r \cdot r=r$, then $r$ is one of preradicals $r_{\alpha}$. Therefore $r \geq \wedge\left\{r_{\alpha} \in \mathbb{P} \mathbb{R} \mid r_{\alpha} \cdot r=r\right\}$, i.e. $\quad r \geq e(r)$, but $e(r) \geq r$, so $e(r)=r$.

$(\Leftarrow)$ Let $e(r)=r$. Then $r \cdot r=e(r) \cdot r$. Since $e(r) \cdot r=r$, so $r \cdot r=r$, i.e. $r$ is idempotent. 
Moreover, since $r \leq r \wedge s \leq e(r)$, if $r$ is idempotent, then $r \wedge s=r$.

Proposition 2.15. Let $r, s \in \mathbb{P}$ and $s$ be an idempotent preradical. Then:

1) $r \% s \leq s$ with $r \leq s$;

2) $(r \wedge s) \cdot s=r \wedge . s$ with $r \leq s$;

3) $(r \% s) \wedge s=r \wedge s$ with $r \leq s$;

4) $(r \cdot s) \wedge . s=r \cdot s$.

Proof. 1) The condition $r \leq s$ ensures the existence of the left quotient $r \wedge s$.

Let $r \leq s$. From the monotony of the left quotient $r \% s \leq s \% s$. If $s$ is idempotent, then $r \% s \leq s$.

2) The condition $r \leq s$ ensures the existence of the left quotient $r \wedge s$.

If $s$ is an idempotent preradical, then $(r \% s) \cdot s=(r \% s) \cdot e(s)$. By Proposition 2.12 we obtain $(r \% s) \cdot s=r \% s$.

3) The condition $r \leq s$ ensures the existence of the left quotient $r \% s$. Moreover, from 1) $r \wedge s \leq s$, which implies the existence of the left quotient $(r \% s) \% s$.

From Proposition 2.7(1) $(r \wedge s) \wedge s=r \wedge(s \cdot s)$. If $s$ is an idempotent preradical, then $(r \% s) \wedge s=r \wedge . s$.

4) From Proposition 2.7(2) $(r \cdot s) \wedge s \leq r \cdot(s \% s)$. If $s$ is an idempotent preradical, then $(r \cdot s) \wedge s \leq r \cdot s$. But from Lemma $2.2(r \cdot s) \wedge s \geq r \cdot s$, therefore $(r \cdot s) \wedge s=r \cdot s$.

The next two statements show when the cancellation properties for the left quotient hold (see Proposition 2.6).

Proposition 2.16. Let $r, s \in \mathbb{P}$. The following conditions are equivalent:

1) $r=(r \cdot s) \wedge s$;

2) $r=t \% s$ for some preradical $t \leq s$.

Proof. The condition $t \leq s$ ensures the existence of the left quotient $t \% s$.

1) $\Rightarrow 2$ ) If $r=(r \cdot s) \% s$, then $r=t \% s$ with $t=r \cdot s$.

$2) \Rightarrow 1$ ) Let $r=t \% s$ for some preradical $t \leq s$. By the definition of the left quotient $(t \% s) \cdot s \geq t$. Using the monotony of the left quotient we obtain $[(t \% s) \cdot s] \wedge . s \geq t \wedge . s$. But from Proposition $2.6[(t \% s) \cdot s] \% . s \leq t \% . s$, thus $[(t \wedge . s) \cdot s] \wedge . s=t \% s$. Since $t \wedge s=r$, we have $(r \cdot s) \wedge . s=r$.

Proposition 2.17. Let $r, s \in \mathbb{P}$. The following conditions are equivalent:

1) $r=(r \% s) \cdot s$ with $r \leq s$;

2) $r=t \cdot s$ for some preradical $t \in \mathbb{P} \mathbb{R}$. 
Proof. The condition $r \leq s$ ensures the existence of the left quotient $r \% s$.

1) $\Rightarrow 2)$ If $r=(r \wedge s) \cdot s$, then $r=t \cdot s$ with $t=r \% s$.

$2) \Rightarrow 1$ ) Let $r=t \cdot s$ for some preradical $t \in \mathbb{P R}$. By Proposition 2.6 $(t \cdot s) \% s \leq t$. Applying the monotony of product we obtain $[(t \cdot s) \% \cdot s] \cdot s \leq$ $t \cdot s$. But from the definition of the left quotient $[(t \cdot s) \% s] \cdot s \geq t \cdot s$, therefore $[(t \cdot s) \% \cdot s] \cdot s=t \cdot s$. Since $t \cdot s=r$, we have $(r \% s) \cdot s=r$.

Now we will study the behaviour of the left quotient $r \% s$ in the case of such types of preradicals as coprime, $\vee$-coprime and coirreducible (see Section $1)$.

Proposition 2.18. If $r$ is a coprime preradical, then the preradical $r \% s$ is coprime for any preradical $s \geq r$.

Proof. The condition $r \leq s$ ensures the existence of the left quotient $r \% s$.

Let the preradical $r \neq 0$ be coprime and $t_{1} \# t_{2} \geq r \% s$ for some preradicals $t_{1}, t_{2} \in \mathbb{P R}$. Using Proposition 2.5 we obtain $r \leq\left(t_{1} \# t_{2}\right) \cdot s$. From Lemma 1.3(2) $\left(t_{1} \# t_{2}\right) \cdot s \leq\left(t_{1} \cdot s\right) \#\left(t_{2} \cdot s\right)$, so $r \leq\left(t_{1} \cdot s\right) \#\left(t_{2} \cdot s\right)$. Since $r$ is coprime, it follows that $r \leq t_{1} \cdot s$ or $r \leq t_{2} \cdot s$. Applying Proposition 2.5 we obtain $r \% s \leq t_{1}$ or $r \% s \leq t_{2}$. So for every $t_{1}, t_{2} \in \mathbb{P R}$ with $t_{1} \# t_{2} \geq r \% s$ we have $t_{1} \geq r \% s$ or $t_{2} \geq r \% s$, which means that the preradical $r \wedge s$ is coprime.

Proposition 2.19. If the preradical $r$ is $\vee$-coprime, then the preradical $r \% s$ is $\vee$-coprime for any preradical $s \geq r$.

Proof. The condition $r \leq s$ ensures the existence of the left quotient $r \% s$.

Let $r$ be $\vee$-coprime and $t_{1} \vee t_{2} \geq r \% s$, for some preradicals $t_{1}, t_{2} \in \mathbb{P}$. Applying Proposition 2.5 we obtain $r \leq\left(t_{1} \vee t_{2}\right) \cdot s$. From the distributivity of product of preradicals relative to join $r \leq\left(t_{1} \cdot s\right) \vee\left(t_{2} \cdot s\right)$. Since $r$ is $\vee$-coprime it follows that $r \leq t_{1} \cdot s$ or $r \leq t_{2} \cdot s$. From Proposition 2.5 we obtain $r \% s \leq t_{1}$ or $r \% s \leq t_{2}$. So for every preradicals $t_{1}, t_{2} \in \mathbb{P} \mathbb{R}$ with $t_{1} \vee t_{2} \geq r \% s$ we have $t_{1} \geq r \% s$ or $t_{2} \geq r \% s$, which means that the preradical $r \% s$ is $\vee$-coprime.

Proposition 2.20. Let $r, s \in \mathbb{P R}$ and $r=t \cdot s$ for some preradical $t \in \mathbb{P R}$. If the preradical $r$ is coirreducible, then the preradical $r \% s$ is coirreducible.

Proof. By the condition $r=t \cdot s$ we have $r \leq s$, which ensures the existence of the left quotient $r \% s$.

Let $r$ be coirreducible and $r \% s=t_{1} \vee t_{2}$ for some preradicals $t_{1}, t_{2} \in \mathbb{P R}$. If $r=t \cdot s$ for some preradical $t$, then by Proposition $2.17 r=(r \% s) \cdot s$, thus $r=\left(t_{1} \vee t_{2}\right) \cdot s$. From the distributivity of product of preradicals 
relative to join $r=\left(t_{1} \cdot s\right) \vee\left(t_{2} \cdot s\right)$. Since $r$ is coirreducible it follows that $t_{1} \cdot s=r$ or $t_{2} \cdot s=r$.

If $t_{1} \cdot s=r$, then from Proposition 2.5 we have $t_{1} \geq r \% s$. But $t_{1} \leq t_{1} \vee t_{2}=r \% s$, therefore $t_{1}=r \% s$.

If $t_{2} \cdot s=r$, then similarly we obtain $t_{2}=r \wedge s$.

So for every preradicals $t_{1}, t_{2} \in \mathbb{P R}$ with $t_{1} \vee t_{2}=r \% s$ we have $t_{1}=r \% s$ or $t_{2}=r \% s$, which means that the preradical $r \% s$ is coirreducible. $([7])$.

Moreover, if the preradical $r$ is coprime, then its equalizer $e(r)$ is coprime

The operation of the left quotient with respect to meet implies some order relations between the associated preradicals.

Corollary 2.21. 1) For every preradicals $r, s \in \mathbb{P} \mathbb{R}$ with $r \leq s$ the following relations hold:

$$
r \cdot s \leq(r \cdot s) \% s \leq r \leq(r \% s) \cdot s \leq r \% s
$$

2) If the preradical $s$ is idempotent, then

$$
r \cdot s=(r \cdot s) \% s \leq r \leq(r \% s) \cdot s=r \% s \leq s
$$

for every preradical $r \leq s$.

\section{Left coquotient with respect to join}

In this section the similar questions are discussed as in the preceding one for the inverse operation of coproduct with respect to join in the class of preradicals $\mathbb{P R}$ of category $R$-Mod.

Definition 3.1. Let $r, s \in \mathbb{P}$. The left coquotient with respect to join of $r$ by $s$ is defined as the greatest preradical among $r_{\alpha} \in \mathbb{P} \mathbb{R}$ with the property $r_{\alpha} \# s \leq r$. We denote this preradical by $r^{\vee / \#} s$.

We will call $r$ the numerator and $s$ the denominator of the left coquotient $r \vee / \# s$.

The following statement is the answer to the question on the existence of the left coquotient with respect to join.

Lemma 3.1. Let $r, s \in \mathbb{P} \mathbb{R}$. The left coquotient $r^{\vee / \#} s$ of $r$ by $s$ with respect to join exists if and only if $r \geq s$ and it can be presented in the form $r^{\vee} / \# s=\vee\left\{r_{\alpha} \in \mathbb{P} \mathbb{R} \mid r_{\alpha} \# s \leq r\right\}$.

Proof. $(\Rightarrow)$ Let there exists the left coquotient $r \vee / \# s$. Then $\exists r_{\alpha} \in \mathbb{P} \mathbb{R}$ such that $r_{\alpha} \# s \leq r$. Since $0 \leq r_{\alpha}$, from the monotony of coproduct of preradicals we obtain $0 \# s \leq r_{\alpha} \# s$, i.e. $s \leq r_{\alpha} \# s$, therefore $s \leq r$. 
$(\Leftarrow)$ Let $r \geq s$. Then $0 \# s=s \leq r$, therefore the family of preradicals $\left\{r_{\alpha} \in \mathbb{P} \mathbb{R} \mid r_{\alpha} \# s \leq r\right\}$ is not empty. So we can consider the preradical $\vee\left\{r_{\alpha} \in \mathbb{P} \mid r_{\alpha} \# s \leq r\right\}$. Using the distributivity of coproduct relative to join of preradicals we have $\left(\underset{r_{\alpha} \# s \leq r}{\vee} r_{\alpha}\right) \# s=\underset{r_{\alpha} \# s \leq r}{\vee}\left(r_{\alpha} \# s\right)$, but since $r_{\alpha} \# s \leq r$ for every preradical $r_{\alpha}$ it follows that $\underset{r_{\alpha} \# s \leq r}{\vee}\left(r_{\alpha} \# s\right) \leq r$, i.e. $\left(\underset{r_{\alpha} \# s \leq r}{\vee} r_{\alpha}\right) \# s \leq r$. By the construction of this preradical is clear that it is the greatest preradical of $\mathbb{P R}$ with the property $r_{\alpha} \# s \leq r$. Therefore $\vee\left\{r_{\alpha} \in \mathbb{P} \mathbb{R} \mid r_{\alpha} \# s \leq r\right\}=r^{\vee} / \# s$.

Moreover, by the proof of Lemma 3.1 we have that $\left(r^{\vee} / \# s\right) \# s \leq r$.

Lemma 3.2. For every $r, s \in \mathbb{P}$ with $r \geq s$ we have $r v_{/ \#} s \leq r$.

Proof. The condition $r \geq s$ ensures the existence of the left coquotient $r v^{\vee} s$. Since $r^{\vee} / \# s \leq\left(r^{\vee} / \# s\right) \# s$, by the definition of the left coquotient we obtain that $r \vee / \# s \leq r$.

Now we show the behaviour of the left coquotient relative to the partial order $(\leq)$ of the class $\mathbb{P R}$.

Proposition 3.3. (Monotony in the numerator) Let $r_{1}, r_{2} \in \mathbb{P R}$ and $r_{1} \leq r_{2}$. Then for every preradical $s \leq r_{1}$ we have $r_{1} \vee_{/ \#} s \leq r_{2} v_{\#} s$.

Proof. Since $s \leq r_{1} \leq r_{2}$, from Lemma 3.1 there exist the left coquotients $r_{1} \vee / \# s, \quad r_{2} v_{\#} s$ and $r_{1} \vee / \# s=\vee\left\{r_{\alpha} \in \mathbb{P} \mid r_{\alpha} \# s \leq r_{1}\right\}, \quad r_{2} \vee / \#=$ $\vee\left\{r_{\beta}^{\prime} \in \mathbb{P} \mathbb{R} \mid r_{\beta}^{\prime} \# s \leq r_{2}\right\}$.

The relations $r_{1} \leq r_{2}$ and $r_{\alpha} \# s \leq r_{1}$ imply $r_{\alpha} \# s \leq r_{2}$, so each $r_{\alpha}$ is one of preradicals $r_{\beta}^{\prime}$, from where it follows that $\vee\left\{r_{\alpha} \in \mathbb{P R} \mid r_{\alpha} \# s \leq r_{1}\right\} \leq$ $\vee\left\{r_{\beta}^{\prime} \in \mathbb{P} \mathbb{R} \mid r_{\beta}^{\prime} \# s \leq r_{2}\right\}$, so $r_{1} \vee / \# s \leq r_{2} \vee / \# s$.

Proposition 3.4. (Antimonotony in the denominator) Let $s_{1}, s_{2} \in \mathbb{P} \mathbb{R}$ and $s_{1} \leq s_{2}$. Then for every preradical $r \geq s_{2}$ we have $r v_{/ \#} s_{1} \geq r^{\vee} / \# s_{2}$.

Proof. Since $r \geq s_{2} \geq s_{1}$ from Lemma 3.1 there exist the left coquotient $r^{\vee / \#} s_{1}, \quad r \vee / \# s_{2} \quad$ and $\quad r \vee / \# s_{1}=\vee\left\{r_{\alpha} \in \mathbb{P} \mathbb{R} \mid r_{\alpha \#} s_{1} \leq r\right\} \quad$ and $\quad r^{\vee / \#} s_{2}=$ $\vee\left\{r_{\beta}^{\prime} \in \mathbb{P} \mathbb{R} \mid r_{\beta}^{\prime} \# s_{2} \leq r\right\}$.

Let $s_{1} \leq s_{2}$, from the monotony of coproduct of preradicals we have $r_{\beta}^{\prime} \# s_{1} \leq r_{\beta}^{\prime} \# s_{2}$, but if $r_{\beta}^{\prime} \# s_{2} \leq r$, then $r_{\beta}^{\prime} \# s_{1} \leq r$. Therefore each preradical $r_{\beta}^{\prime}$ is one of preradicals $r_{\alpha}$, which implies that $\vee\left\{r_{\beta}^{\prime} \in \mathbb{P} \mathbb{R} \mid r_{\beta}^{\prime} \# s_{2} \leq r\right\} \leq$ $\vee\left\{r_{\alpha} \in \mathbb{P} \mathbb{R} \mid r_{\alpha} \# s_{1} \leq r\right\}$, i.e. $\quad r^{\vee} / \# s_{2} \leq r^{\vee} / \# s_{1}$. 
The next statement is useful for applications.

Proposition 3.5. Let $r, s \in \mathbb{P} \mathbb{R}$ and $r \geq s$. Then for every preradical $t \in \mathbb{P} \mathbb{R}$ we have:

$$
r \geq t \# s \Leftrightarrow r \vee / \# s \geq t
$$

Proof. From Lemma 3.1 there exists the left coquotient $r \vee / \# s$ and $r^{\vee} / \# s=$ $\vee\left\{r_{\alpha} \in \mathbb{P} \mathbb{R} \mid r_{\alpha} \# s \leq r\right\}$.

$(\Rightarrow)$ Let $t \# s \leq r$. Then $t$ is one of preradicals $r_{\alpha}$, hence $t \leq$ $\vee\left\{r_{\alpha} \in \mathbb{P} \mathbb{R} \mid r_{\alpha} \# s \leq r\right\}=r^{\vee} / \# s$.

$(\Leftarrow)$ Let $t \leq r^{\vee} / \# s$. From the monotony of coproduct $t \# s \leq\left(r^{\vee} / \# s\right) \# s$. By the definition of the left coquotient we have $\left(r^{\vee} / \# s\right) \# s \leq r$, thus $t \# s \leq$ $r$.

In the following statements some properties of the left coquotient are indicated.

Proposition 3.6. For every preradicals $r, s \in \mathbb{P}$ we have:

$$
(r \# s) \vee / \# s \geq r .
$$

Proof. Since $r \# s \geq s$, from Lemma 3.1 there exists the left coquotient $(r \# s) v^{\vee} / \# s$ and $(r \# s) \vee / \# s=\vee\left\{r_{\alpha} \in \mathbb{P} \mathbb{R} \mid r_{\alpha} \# s \leq r \# s\right\}$.

Since $r \# s \leq r \# s$, we have that the preradical $r$ is one of preradicals $r_{\alpha}$, therefore $r \leq \vee\left\{r_{\alpha} \in \mathbb{P} \mathbb{R} \mid r_{\alpha} \# s \leq r \# s\right\}$, i.e. $r \leq(r \# s) v^{\vee} / \#$.

Proposition 3.7. Let $r, s, t \in \mathbb{P} \mathbb{R}$. The following relations are true:

1) $(r \vee / \# s) \vee_{/ \#} t=r v^{\vee}(t \# s)$ with $r \geq t \# s$;

2) $(r \# s) \vee / \# t \geq r \#(s \vee / \# t)$ with $s \geq t$.

Proof. 1) If $r \geq t \# s$, then there exists the left coquotient $r^{\vee / \#}(t \# s)$. In this case, since $t \# s \geq s$ we have $r \geq s$, so there exists the left coquotient $r v^{\vee} s$. Moreover, by Proposition $3.5 t_{\#} s \leq r \Leftrightarrow r v^{\vee} s \geq t$, which ensures the existence of the left coquotient $\left(r v_{/ \#} s\right) \bar{v}_{/ \#} t$. From Lemma 3.1 we have $r^{\vee} / \# s=\vee\left\{r_{\alpha} \in \mathbb{P} R \mid r_{\alpha} \# s \leq r\right\}, r \vee / \#(t \# s)=\vee\left\{s_{\beta} \in \mathbb{P} \mathbb{R} \mid s_{\beta} \#(t \# s) \leq r\right\}$ and $(r \vee / \# s) \vee / \# t=\vee\left\{t_{\gamma} \in \mathbb{P} R \mid t_{\gamma} \# t \leq r \vee / \# s\right\}$.

$(\leq)$ Let $t_{\gamma} \# t \leq r^{\vee} / \# s$. Using the monotony of coproduct of preradicals we obtain $\left(t_{\gamma} \# t\right) \# s \leq(r \vee / \# s) \# s$, but from the definition of the left coquotient $\left(r^{\vee} / \# s\right) \# s \leq r$, so $\left(t_{\gamma} \# t\right) \# s \leq r$. By the associativity of coproduct of preradicals we have $t_{\gamma} \#(t \# s) \leq r$, which means that each preradical $t_{\gamma}$ is one of preradicals $s_{\beta}$, therefore $\vee\left\{t_{\gamma} \in \mathbb{P} \mathbb{R} \mid t_{\gamma} \# t \leq r r^{\vee} s\right\} \leq$ $\vee\left\{s_{\beta} \in \mathbb{P} \mathbb{R} \mid s_{\beta} \#(t \# s) \leq r\right\}$, i.e $\left(r^{\vee} / \# s\right) \vee / \# t \leq r \vee / \#(t \# s)$. 
$(\geq)$ Let $s_{\beta} \#(t \# s) \leq r$. Since $\left(s_{\beta} \# t\right) \# s=s_{\beta} \#(t \# s)$ we have $\left(s_{\beta} \# t\right) \# s \leq r$, but $r^{\vee} / \# s$ is the greatest preradical among $r_{\alpha} \in \mathbb{P} \mathbb{R}$ with the property $r_{\alpha} \# s \leq r$, so $s_{\beta} \# t \leq r^{\vee} / \# s$, which means that $s_{\beta}$ is one of preradicals $t_{\gamma}$. Thus $\vee\left\{s_{\beta} \in \mathbb{P} \mathbb{R} \mid s_{\beta} \#(t \# s) \leq r\right\} \leq \vee\left\{t_{\gamma} \in \mathbb{P} \mathbb{R} \mid t_{\gamma} \# t \leq r \vee / \# s\right\}$, i.e. $\quad r v_{/ \#}(t \# s) \leq\left(r^{\vee} / \# s\right) v_{/ \#} t$.

2) The condition $s \geq t$ ensures the existence of the left coquotient $s^{v} / \# t$. Moreover, since $r \# s \geq s$ we have $r \# s \geq t$, so there exists the left coquotient $(r \# s) \vee / \# t$.

By the definition of the left coquotient $s \geq\left(s^{\vee} / \# t\right) \# t$, from the monotony and the property of associativity of coproduct we obtain $r \# s \geq r \#\left[\left(s^{\vee} / \# t\right) \# t\right]=$ $\left[r \#\left(s^{\vee} / \# t\right)\right] \# t$. Applying Proposition 3.5 we have $(r \# s){ }^{\vee} / \# t \geq r \#\left(s^{\vee} / \# t\right)$.

Proposition 3.8. Let $r, s, t \in \mathbb{P}$ and $r \geq s$. The following relations hold:

1) $\left(r^{\vee / \#} t\right)_{/ \#}^{\vee}\left(s^{\vee} / \# t\right) \geq r^{\vee} / \# s$ or $\left(r^{\vee} / \# s\right) \#\left(s^{\vee} / \# t\right) \leq r^{\vee} / \#$ for every preradical $t \leq s$;

2) $(r \# t)^{\vee / \#}(s \# t) \geq r^{\vee / \#} s$ for every preradical $t \in \mathbb{P}$.

Proof. 1) The condition $r \geq s$ ensures the existence of the left coquotient $r \vee / \# s$. In the this case if $t \leq s$, then $r \geq t$, so there exist the left coquotients $s \vee_{\#} t$ and $r \vee / \# t$. Moreover, since $r \geq s$, from the monotony of the left coquotient we have $r v^{\vee} t \geq s^{\vee} / \#$, which ensures the existence of the left coquotien $(r \vee / \# t) \vee / \#(s \vee / \# t)$.

From Proposition 3.5 we have

$\left(r v_{/ \#} t\right)^{\vee / \#}\left(s^{\vee} / \# t\right) \geq r^{\vee} / \# s \Leftrightarrow\left(r^{\vee} / \# s\right) \#\left(s^{\vee} / \# t\right) \leq r^{\vee} / \# t$.

By the definition of the left coquotient $r \geq(r \vee / \# s) \# s$ and $s \geq\left(s^{\vee / \#} t\right) \# t$. Using the monotony and the property of associativity of coproduct we obtain $r \geq(r \vee / \# s) \# s \geq(r \vee / \# s) \#\left[\left(s^{\vee} / \# t\right) \# t\right]=\left[(r \vee / \# s) \#\left(s^{\vee} / \# t\right)\right] \# t$. Applying Proposition 3.5 we have $r \vee / \# t \geq(r \vee / \# s) \#(s \vee / \# t)$.

2) The condition $r \geq s$ ensures the existence of the left coquotient $r \vee / \# s$. Moreover, from the monotony of coproduct we have $r \# t \geq s \# t$ for every preradical $t \in \mathbb{P}$, which implies the existence of the left coquotient $(r \# t) \vee / \#(s \# t)$.

From Proposition 3.5 the relation of this statement is equivalent to the relation $r \# t \geq(r \vee / \# s) \#(s \# t)$.

By the definition of the left coquotient $r \geq\left(r v_{/ \#} s\right) \# s$, using the monotony and the property of associativity of coproduct of preradicals we obtain $r \# t \geq$ $\left[\left(r^{\vee} / \# s\right) \# s\right] \# t=\left(r^{\vee} / \# s\right) \#(s \# t)$.

The following two statements show the relation between the left coquotient with respect to join and the lattice operations of $\mathbb{P R}$. 
Proposition 3.9. (The left distributivity of the left coquotient $r \vee / \# s$ relative to meet) For any preradical $s \in \mathbb{P} \mathbb{R}$ and for any family of preradicals $\left\{r_{\alpha} \in \mathbb{P R} \mid r_{\alpha} \geq s, \alpha \in \mathfrak{A}\right\}$ the following relation holds:

$$
\left(\wedge_{\alpha \in \mathfrak{A}} r_{\alpha}\right) v_{/ \#} s=\wedge_{\alpha \in \mathfrak{A}}\left(r_{\alpha} v_{/ \#} s\right) \text {. }
$$

Proof. The relations $r_{\alpha} \geq s, \alpha \in \mathfrak{A}$ ensures the existence of the left coquotients $r_{\alpha} \vee^{\vee} s, \alpha \in \mathfrak{A}$. But in this case $\underset{\alpha \in \mathfrak{A}}{\wedge} r_{\alpha} \geq s$, so there exists the left coquotient $\left(\underset{\alpha \in \mathfrak{A}}{\wedge} r_{\alpha}\right) v_{/ \#} s$.

$(\leq)$ From Lemma $3.1 \quad\left({ }_{\alpha \in \mathfrak{A}} r_{\alpha}\right) \vee_{/ \#} s=\vee\left\{t_{\beta} \in \mathbb{P R} \mid t_{\beta} \# s \leq \underset{\alpha \in \mathfrak{A}}{\wedge_{\alpha}} r_{\alpha}\right\}$ and $r_{\alpha} \vee_{/ \#} s=\vee\left\{r_{\gamma}^{\prime} \in \mathbb{P} \mid r_{\gamma}^{\prime} \# s \leq r_{\alpha}\right\}$.

Let $t_{\beta} \# s \leq \bigwedge_{\alpha \in \mathfrak{A}}^{\wedge} r_{\alpha}$. Since $\underset{\alpha \in \mathfrak{A}}{\wedge} r_{\alpha} \leq r_{\alpha}$ for every $\alpha \in \mathfrak{A}$ we have $t_{\beta} \# s \leq$ $r_{\alpha}$, which means that each preradical $t_{\beta}$ is one of preradicals $r_{\gamma}^{\prime}$. This implies $\vee\left\{t_{\beta} \in \mathbb{P R} \mid t_{\beta} \# s \leq \underset{\alpha \in \mathfrak{A}}{\wedge} r_{\alpha}\right\} \leq \vee\left\{r_{\gamma}^{\prime} \in \mathbb{P R} \mid r_{\gamma}^{\prime} \# s \leq r_{\alpha}\right\}$ for every $\alpha \in \mathfrak{A}$, therefore $\vee\left\{t_{\beta} \in \mathbb{P} \mathbb{R} \mid t_{\beta} \# s \leq \wedge_{\alpha \in \mathfrak{A}} r_{\alpha}\right\} \leq \wedge_{\alpha \in \mathfrak{A}}\left(\vee\left\{r_{\gamma}^{\prime} \in \mathbb{P R} \mid r_{\gamma}^{\prime} \# s \leq r_{\alpha}\right\}\right)$, i.e. $\left(\hat{\alpha \in \mathfrak{A}}_{\alpha} r_{\alpha}\right) v_{/ \#} s \leq \wedge_{\alpha \in \mathfrak{A}}\left(r_{\alpha} v_{\| \mathbb{H}} s\right)$.

$(\geq)$ By the definition of the left coquotient we have $r_{\alpha} \geq\left(r_{\alpha} \vee / \# s\right) \# s$ for every $\alpha \in \mathfrak{A}$, which implies that $\underset{\alpha \in \mathfrak{A}}{\wedge} r_{\alpha} \geq \underset{\alpha \in \mathfrak{A}}{\wedge}\left[\left(r_{\alpha} \vee / \# s\right) \# s\right]$. From the distributivity of coproduct of preradicals relative to meet it follows that $\underset{\alpha \in \mathfrak{A}}{\wedge} r_{\alpha} \geq$ $\left[\wedge_{\alpha \in \mathfrak{A}}\left(r_{\alpha} \vee / \# s\right)\right] \# s$. Using Proposition 3.5 we obtain that $\left(\hat{\alpha \in \mathfrak{A}}^{\wedge} r_{\alpha}\right)^{\alpha \in \mathfrak{A}} v^{\vee} s \geq$ $\widehat{\Lambda}_{\alpha \in \mathfrak{A}}\left(r_{\alpha}{ }^{\vee / \#} s\right)$.

Proposition 3.10. In the class $\mathbb{P R}$ the following relations are true:
1) $\left(\underset{\alpha \in \mathfrak{A}}{\vee} r_{\alpha}\right) \vee / \# s \geq \underset{\alpha \in \mathfrak{A}}{\vee}\left(r_{\alpha} \vee_{/ \#} s\right)$ for $r_{\alpha} \geq s, \alpha \in \mathfrak{A}$;
2) $r \vee / \#\left(\underset{\alpha \in \mathfrak{A}}{\wedge} s_{\alpha}\right) \geq \underset{\alpha \in \mathfrak{A}}{\vee}\left(r^{\vee} / \# s_{\alpha}\right)$ for $r \geq s_{\alpha}, \alpha \in \mathfrak{A}$;
3) $r \vee / \#\left(\underset{\alpha \in \mathfrak{A}}{\vee} s_{\alpha}\right) \leq \wedge_{\alpha \in \mathfrak{A}}\left(r^{\vee / \#} s_{\alpha}\right)$ for $r \geq s_{\alpha}, \alpha \in \mathfrak{A}$.

Proof. 1) The conditions $r_{\alpha} \geq s, \alpha \in \mathfrak{A}$ ensure the existence of the left coquotients $r_{\alpha} \vee_{/ \#} s, \alpha \in \mathfrak{A}$. In this case $\underset{\alpha \in \mathfrak{A}}{\vee} r_{\alpha} \geq s$, so there exists the left 
coquotient $\left(\underset{\alpha \in \mathfrak{A}}{\vee} r_{\alpha}\right) v_{/ \#} s$.

By the definition of the left coquotient we have $r_{\alpha} \geq\left(r_{\alpha} \mathrm{v}_{\|} s\right) \# s$ for every $\alpha \in \mathfrak{A}$, which implies that $\underset{\alpha \in \mathfrak{A}}{\vee} r_{\alpha} \geq \underset{\alpha \in \mathfrak{A}}{\vee}\left[\left(r_{\alpha} \vee^{\vee} s\right) \# s\right]$. From the distributivity of coproduct of preradicals relative to join it follows that $\underset{\alpha \in \mathfrak{A}}{\vee} r_{\alpha} \geq$ $\left[\vee_{\alpha \in \mathfrak{A}}\left(r_{\alpha} \vee_{/ \#} s\right)\right] \# s$. Applying Proposition 3.5 we obtain $\left(\vee_{\alpha \in \mathfrak{A}} r_{\alpha}\right)^{\vee \in / \#} s \geq$ $\vee_{\alpha \in \mathfrak{A}}\left(r_{\alpha} \vee / \# s\right)$.

2) The conditions $r \geq s_{\alpha}, \alpha \in \mathfrak{A}$ ensure the existence of the left coquotients $r^{\vee} / \# s_{\alpha}, \alpha \in \mathfrak{A}$. Moreover, in this case $r \geq \wedge_{\alpha \in \mathfrak{A}} s_{\alpha}$, which implies the existence of the left coquotient $r^{\vee} / \#\left(\bigwedge_{\alpha \in \mathfrak{A}}^{\wedge} s_{\alpha}\right)$.

Since $\wedge_{\alpha \in \mathfrak{A}} s_{\alpha} \leq s_{\alpha}$ for every $\alpha \in \mathfrak{A}$, from Proposition 3.4 we have

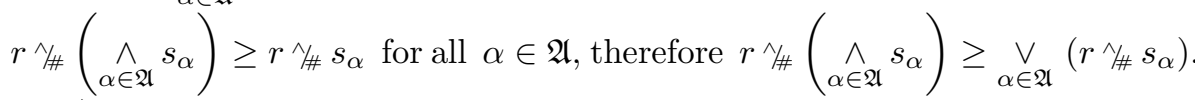

3) The conditions $r \geq s_{\alpha}, \alpha \in \mathfrak{A}$ ensure the existence of the left coquotients $r^{\vee} / \mathbb{\#} s_{\alpha}, \alpha \in \mathfrak{A}$. In this case $r \geq \underset{\alpha \in \mathfrak{A}}{\vee} s_{\alpha}$, which implies the existence of the left coquotient $r \vee / \#\left(\underset{\alpha \in \mathfrak{A}}{\vee} s_{\alpha}\right)$.

For every $\alpha \in \mathfrak{A}$ we have $\underset{\alpha \in \mathfrak{A}}{\vee} s_{\alpha} \geq s_{\alpha}$. Using the antimonotony in the denominator of the left coquotient it follows that $r^{\vee} / \#\left(\underset{\alpha \in \mathfrak{A}}{\vee} s_{\alpha}\right) \leq r^{\vee / \#} s_{\alpha}$ for all $\alpha \in \mathfrak{A}$, therefore $r^{\vee} / \#\left(\vee_{\alpha \in \mathfrak{A}} s_{\alpha}\right) \leq \hat{\Lambda \in \mathfrak{A}}\left(r^{\vee} / \# s_{\alpha}\right)$.

In continuation we study some particular cases of the left coquotient with respect to join.

Proposition 3.11. Let $r, s \in \mathbb{P}$. Then:

1) $r \vee / \# r=c(r)$ (see Definition 1.2);

2) $r \vee / \# 0=r$

3) $1 \vee / \# s=1$.

Proof. From the definition of the left coquotient we obtain:

1) $r^{\vee / \#} r=\vee\left\{r_{\alpha} \in \mathbb{P} \mathbb{R} \mid r_{\alpha} \# r \leq r\right\}=\vee\left\{r_{\alpha} \in \mathbb{P} \mathbb{R} \mid r_{\alpha} \# r=r\right\}=c(r)$;

2) $r \vee / \# 0=\vee\left\{r_{\alpha} \in \mathbb{P} \mid r_{\alpha} \# 0 \leq r\right\}=\vee\left\{r_{\alpha} \in \mathbb{P} \mathbb{R} \mid r_{\alpha} \leq r\right\}=r$;

3) $1 \vee / \# s=\vee\left\{r_{\alpha} \in \mathbb{P} \mathbb{R} \mid r_{\alpha} \# s \leq 1\right\}=\vee\left\{r_{\alpha} \mid r_{\alpha} \in \mathbb{P} \mathbb{R}\right\}=1$. 
By Propositions 3.11 we have the following particular cases:
(1) $0 \vee / \# 0=0$;
(2) $1 \mathrm{v} / \# 1=1$.

Applying to the relation $r \geq s \geq 0$ Proposition 3.4 we obtain that $r \vee / \# \leq r \vee / \# s \leq r \vee / \#$ 0, i.e. $c(r) \leq r \vee / \# s \leq r$.

Moreover, from the distributivity of product of preradicals relative to join we obtain $c(r) \# r=\left(\underset{r_{\alpha} \# r=r}{\vee} r_{\alpha}\right) \# r=\underset{r_{\alpha} \# r=r}{\vee}\left(r_{\alpha} \# r\right)=r$ for every $r \in \mathbb{P R}$.

The following statement shows some properties of the co-equalizer.

Proposition 3.12. If $r, s \in \mathbb{P}$ and $r \geq s$, then:

1) $c(r) \#(r \vee / \# s)=r \vee / \# s$;

2) $(r \vee / \# s) \# c(s)=r^{\vee / \#} s$;

3) $(r \vee / \# s) \vee / \# c(s)=r \vee / \# s$.

Proof. The condition $r \geq s$ ensures the existence of the left coquotient $r \vee / \# s$.

1) $c(r) \#(r \vee / \# s)=(r \vee / \# r) \#(r \vee / \# s)$. From Proposition 3.8(1) we have $\left(r^{\vee / \#} r\right) \#\left(r^{\vee} / \# s\right) \leq r^{\vee} / \# s$, but $\left(r^{\vee} / \# r\right) \#\left(r^{\vee} / \# s\right) \geq r^{\vee} / \# s$, so $c(r) \#\left(r^{\vee} / \# s\right)=$ $r \vee / \# s$.

2) $\left(r^{\vee} / \# s\right) \# c(s)=\left(r^{\vee} / \# s\right) \#\left(s^{\vee} / \# s\right)$. From Proposition 3.8(1) we have $(r \vee / \# s) \#\left(s^{\vee} / \# s\right) \leq r \vee / \# s$, but $(r \vee / \# s) \#\left(s^{\vee} / / \# s\right) \geq r^{\vee} / / \#$, so $(r \vee / \# s) \# c(s)=$ $r \mathrm{v}^{\mathrm{N}} \mathrm{s}$.

3) $\left(r^{\vee} / \# s\right) v_{/ \#} c(s)=\left(r^{\vee} / \# s\right) v_{/ \#}\left(s v^{\vee} s\right)$. Using Proposition 3.8(1) we obtain

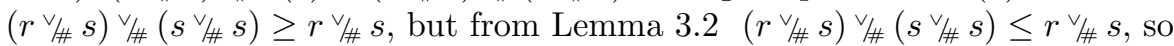
$(r \vee / \# s) \vee / \# c(s)=r \vee / \# s$.

We will now consider the case of radical.

Remark 3.13. ([5]) For every preradical $r \in \mathbb{P}$ we have $c(r)$ is a radical.

Proof. $c(r) \# c(r)=\left(r v_{/ \#} r\right) \#\left(r v_{/ \#} r\right)$. From Proposition 3.8(1) we have that $\left(r \vee_{/ \#} r\right) \#(r \vee / \# r) \leq r v_{/ \#} r$, but $\left(r \vee_{/ \#} r\right) \#\left(r v_{/ \#} r\right) \geq r v_{/ \#} r$, hence $c(r) \# c(r)=$ $c(r)$, i.e. $c(r)$ is a radical.

Proposition 3.14. ([5]) Preradical $r$ is a radical if and only if $c(r)=r$.

Proof. $(\Rightarrow)$ By Definition $1.2 \quad c(r)=\vee\left\{r_{\alpha} \in \mathbb{P} \mathbb{R} \mid r_{\alpha} \# r=r\right\}$. Let $r$ is a radical, i.e. $r \# r=r$, hence the preradical $r$ is one of preradicals $r_{\alpha}$. Therefore $r \leq \vee\left\{r_{\alpha} \in \mathbb{P} \mathbb{R} \mid r_{\alpha} \# r=r\right\}$, i.e. $\quad r \leq c(r)$, but $c(r) \leq r$, so $c(r)=r$.

$(\Leftarrow)$ Let $c(r)=r$. Then $r \# r=c(r) \# r$, but $c(r) \# r=r$, so $r \# r=r$, which means that the preradical $r$ is a radical. 
Moreover, since $c(r) \leq r^{\vee / \#} s \leq r$, if $r$ is a radical, then $r \vee / \# s=r$.

Proposition 3.15. Let $r, s \in \mathbb{P} \mathbb{R}$ and $s$ is a radical. Then:

1) $r \vee / \# s \geq s$ with $r \geq s$;

2) $(r \vee / \# s) \# s=r \vee / \# s$ with $r \geq s$;

3) $(r \vee / \# s) \vee / \# s=r \vee / \# s$ with $r \geq s$;

4) $(r \# s) \vee / \# s=r \# s$.

Proof. 1) The condition $r \geq s$ ensures the existence of the left coquotient $r \vee / \# s$.

Let $r \geq s$. From the monotony of the left coquotient $r^{\vee} / \# s^{\vee} \geq s^{\vee} / \# s$. If $s$ is a radical, then $r \% s \geq s$.

2) The condition $r \geq s$ ensures the existence of the left coquotient $r^{\vee} / \# s$.

If $s$ is a radical, then $(r \vee / \# s) \# s=(r \vee / \# s) \# c(s)$, but Proposition 3.12 $(r \vee / \# s) \# c(s)=r \vee / \# s$, so $\left(r^{\vee} / \# s\right) \# s=r^{\vee} / \# s$.

3) The condition $r \geq s$ ensures the existence of the left coquotient $r \vee / \# s$. Moreover, by 1) $r^{\vee} / \# s \geq s$, which implies the existence of the left coquotient $(r \vee / \# s) \vee / \# s$.

From Proposition 3.7(1) $\left(r^{\vee / \#} s\right)^{\vee / \#} s=r^{\vee} / \#(s \# s)$. If $s$ is a radical, then $(r \vee / \# s) \vee / \# s=r \vee / \# s$.

4) From Proposition 3.7(2) $(r \# s) v_{/ \#} s \geq r \#\left(s^{\vee} / \# s\right)$. If $s$ is a radical, then $(r \# s) \vee / \# s \geq r \# s$. But by Lemma $3.2(r \# s) v_{/ \#} s \leq r \# s$, therefore $(r \# s) \vee / \# s=r \# s$.

In the next two statements it is shown when the cancellation properties for the left coquotient hold (see Proposition 3.6).

Proposition 3.16. Let $r, s \in \mathbb{P}$. The following conditions are equivalent:

1) $r=(r \# s) \vee / \# s$;

2) $r=t^{\vee} / \# s$ for some preradical $t \geq s$.

Proof. The condition $t \geq s$ ensures the existence of the left coquotient $t \vee / \# s$.

$1) \Rightarrow 2)$ Let $r=(r \# s) \vee / \# s$. Then $r=t^{\vee} / \# s$ with $t=r \# s$.

$2) \Rightarrow 1$ ) Let $r=t^{\vee} / \# s$ for some preradical $t \geq s$. By the definition of the left coquotient $\left(t^{\vee} / \# s\right) \# s \leq t$. Applying the monotony of the left coquotient we obtain $\left[\left(t v^{\vee} / \# s\right) \# s\right] \vee_{/ \#} s \leq t v_{/ \#} s$, but from Proposition 3.6 $\left[\left(t^{\vee / \#} s\right) \# s\right]^{\vee / \#} s \geq t t_{/ \#} s$, thus $\left[\left(t^{\vee} / \# s\right) \# s\right]^{\vee / \#} s=t^{\vee} / \# s$. Since $t^{\vee} / \# s=r$ we have $(r \# s) \vee / \# s=r$.

Proposition 3.17. Let $r, s \in \mathbb{P} \mathbb{R}$. The following conditions are equivalent:

1) $r=\left(r^{\vee / \#} s\right) \# s$ with $r \geq s$;

2) $r=t \# s$ for some preradical $t \in \mathbb{P} \mathbb{R}$. 
Proof. The condition $r \geq s$ ensures the existence of the left coquotient $r \vee / \# s$.

1) $\Rightarrow 2)$ Let $r=(r \vee / \# s) \# s$. Then $r=t \# s$ with $t=r \vee / \# s$.

$2) \Rightarrow 1$ ) Let $r=t \# s$ for some preradical $t \in \mathbb{P R}$. By Proposition 3.6 $(t \# s) \vee_{/ \#} s \geq t$. Using the monotony of coproduct we obtain $\left[(t \# s) \vee_{/ \#} s\right] \# s \geq$ $t \# s$, but from the definition of the left coquotient $\left[(t \# s) v_{/ \#} s\right] \# s \leq t \# s$, thus $\left[(t \# s) v_{/ \#} s\right] \# s=t \# s$. Since $t \# s=r$, we have $\left(r^{\vee} / \# s\right) \# s=r$.

In continuation we indicate the behaviour of the left coquotient $r v_{\mathbb{A}} s$ in the case of such types of preradicals as prime, $\wedge$-prime and irreducible.

Proposition 3.18. If $r$ is a prime preradical, then $r^{\vee / \# s}$ is a prime preradical for any preradical $s \leq r$.

Proof. The condition $r \geq s$ ensures the existence of the left coquotient $r \vee / \# s$.

Let the preradical $r \neq 1$ be prime and $t_{1} \cdot t_{2} \leq r^{\vee} / \# s$ for some preradicals $t_{1}, t_{2} \in \mathbb{P R}$. From Proposition 3.5 we have $r \geq\left(t_{1} \cdot t_{2}\right) \# s$. By Lemma 1.3(1) $\left(t_{1} \cdot t_{2}\right) \# s \geq\left(t_{1} \# s\right) \cdot\left(t_{2} \# s\right)$, so $r \geq\left(t_{1} \# s\right) \cdot\left(t_{2} \# s\right)$. Since $r$ is prime, it follows that $r \geq t_{1} \# s$ or $r \geq t_{2} \# s$. Using Proposition 3.5 we obtain $r \vee / \# s \geq t_{1}$ or $r v_{/ \#} s \geq t_{2}$. So for every $t_{1}, t_{2} \in \mathbb{P} \mathbb{R}$ with $t_{1} \cdot t_{2} \leq r \vee / \# s$ we have $t_{1} \leq r \vee / \# s$ or $t_{2} \leq r \vee / \# s$, which means that the preradical $r \vee / \# s$ is prime.

Proposition 3.19. If the preradical $r$ is $\wedge$-prime, then the preradical $r \vee / \# s$ is $\wedge$-prime for any preradical $s \leq r$.

Proof. The condition $r \geq s$ ensures the existence of the left coquotient $r \vee / \# s$.

Let $r$ be $\wedge$-prime and $t_{1} \wedge t_{2} \leq r v_{/ \#} s$ for some preradicals $t_{1}, t_{2} \in \mathbb{P R}$. From Proposition 3.5 we have $r \geq\left(t_{1} \wedge t_{2}\right) \# s$. Using the distributivity of coproduct of preradicals relative to meet we obtain $r \geq\left(t_{1} \# s\right) \wedge\left(t_{2} \# s\right)$. Since $r$ is $\wedge$-prime, it follows that $r \geq t_{1} \# s$ or $r \geq t_{2} \# s$. From Proposition $3.5 r^{\vee / 4} s \geq t_{1}$ or $r \vee_{/ A} s \geq t_{2}$. So for every preradicals $t_{1}, t_{2} \in \mathbb{P R}$ with $t_{1} \wedge t_{2} \leq r \vee / \# s$ we have $t_{1} \leq r \vee / \# s$ or $t_{2} \leq r \vee / \# s$, which means that the preradical $r \vee / \# s$ is $\wedge$-prime.

Proposition 3.20. Let $r, s \in \mathbb{P R}$ and $r=t \# s$ for some preradical $t \in \mathbb{P} \mathbb{R}$. If the preradical $r$ is irreducible, then the preradical $r^{\vee} A s$ is irreducible.

Proof. By the condition $r=t \# s$ we have $r \geq s$, which ensures the existence of the left coquotient $r \vee / \# s$.

Let $r$ be irreducible and $r^{\vee / \#} s=t_{1} \wedge t_{2}$ for some preradicals $t_{1}, t_{2} \in \mathbb{P R}$. If $r=t \# s$ for some preradical $t$, then by Proposition $3.17 r=\left(r^{\vee} / \# s\right) \# s$, so $r=\left(t_{1} \wedge t_{2}\right) \# s$. Using the distributivity of coproduct of preradicals relative to meet we obtain $r=\left(t_{1} \# s\right) \wedge\left(t_{2} \# s\right)$. Since $r$ is irreducible, it follows that $r=t_{1} \# s$ or $r=t_{2} \# s$. From Proposition 3.5 these relations have the 
form $r \vee / \# s \geq t_{1}$ or $r v^{\vee} s \geq t_{2}$ respectively. But $r \vee / \# s=t_{1} \wedge t_{2}$, hence $t_{1} \geq r \vee / \# s$ and $t_{2} \geq r \vee / \# s$. Therefore we obtain $\quad r^{\vee} / \# s=t_{1}$ or $\quad r \vee / \# s=t_{2}$. So for every preradicals $t_{1}, t_{2} \in \mathbb{P} \mathbb{R}$ with $t_{1} \wedge t_{2}=r \vee / \# s$ we have $t_{1}=r \vee / \# s$ or $t_{2}=r^{\vee} / \# s$, which means that the preradical $r^{\vee / \#} s$ is irreducible.

Moreover, if the preradical $r$ is prime, then its co-equalizer $c(r)$ is prime $([6])$.

The operation of the left coquotient with respect to join implies some order relations between the associated preradicals.

Corollary 3.21. 1) For every preradicals $r, s \in \mathbb{P} \mathbb{R}$ with $r \geq s$ the following relations hold:

2) If $s$ is a radical, then:

$$
r \vee / \# s \leq(r \vee / \# s) \# s \leq r \leq(r \# s) \vee^{\vee} / \# s \leq r \# s
$$

$$
s \leq r \vee / \# s=\left(r^{\vee} / \# s\right) \# s \leq r \leq(r \# s) v^{\vee} / \# s=r \# s
$$

for every preradical $r \geq s$.

In conclusion we can say that in the class $\mathbb{P}$ of the category $R$-Mod two new operations are defined and investigated, namely, left quotient with respect to meet and left coquotient with respect to join. These operations are partial in the sense that they do not exist for any two preradicals, but only under certain conditions. They possess a series of properties related with the four operations of the class $\mathbb{P} R$ and are consistent with a series of a notions and constructions from the theory of radicals.

\section{References}

[1] Bican L., Kepka T., Nemec P. Rings, modules and preradicals. Marcel Dekker, New York, 1982.

[2] Kashu A. I. Radicals and torsions in modules. Kishinev, Shtiintsa, 1983 (in Russian).

[3] Guţan M., Guţan C., Radu Gh., Ştefănescu M. Preradicali şi teorii de torsiune. Iaşi, Univ. "Al.I.Cuza", 1986.

[4] Raggi F., Rios J., Rincon H., Fernandes-Alonso R., Signoret C. The lattice structure of preradicals. Commun. Algebra, 30(3), 2002, 1533-1544.

[5] Raggi F., Rios J., Rincon H., Fernandes-Alonso R., Signoret C. The lattice structure of preradicals II. J. of Algebra and its Applications, Vol. 1., No. 2, 2002, 201-214. 
[6] Raggi F., Rios J., Rincon H., Fernandes-Alonso R., Signoret C. Prime and irreducible preradicals. J. of Algebra and its Applications, 2005, Vol. 4, No. 4, 451-466.

[7] Raggi F., Rios J., Wisbauer R. Coprime preradicals and modules. Journal of Pure and Applied Algebra, 2005, 200, 51-69.

[8] Golan J. S. Linear topologies on a ring. Logman Sci. Techn., New York, 1987.

[9] Kashu A. I. On inverse operations in the lattices of submodules. Algebra and Discrete Mathematics, 2012, Vol. 13, No. 2, 273-288.

[10] Kashu A. I. On partial inverse operations in the lattice of submodules. Bul. Acad. Ştiinţe a Repub. Moldova, Mat., 2012, No. 2(69), 59-73.

[11] JARDAN I. On the inverse operations in the class of preradicals of a module category, I. Bul. Acad. Ştiinţe a Repub. Moldova. Mat., 2017, No. $1(83), 57-66$.

[12] JARDAN I. On the inverse operations in the class of preradicals of a module category, II. Bul. Acad. Ştiinţe a Repub. Moldova. Mat., 2017, No. $2(84), 77-87$.

Ion Jardan,

Technical University of Moldova,

Institute of Mathematics and Computer Science, Republic of Moldova,

str. Academiei 5, Chişinău, MD-2028 Moldova.

Email: ion.jardan@mate.utm.md 